\title{
Characterization of Clinical and CT Manifestations of Pulmonary Cryptococcosis with Consolidation
}

\author{
Dengfa Yang, $\mathrm{MM}^{1}$; Lianhua Yu, BSc ${ }^{2}$; Jianhua Luo, $\mathrm{MM}^{3}$; Jianmin Shen, $\mathrm{BSc}^{{ }^{*}}$; Dongguo Wang, $\mathrm{BSc}^{4^{*}}$; Pingding Kuang, $\mathrm{MD}^{5}$; \\ Gangzhe Fu, $\mathrm{MM}^{6}$ \\ 'Department of Radiology, Taizhou Municipal Hospital, Taizhou, Zhejiang, China \\ ${ }^{2}$ Department of Laboratory Medicine, Taizhou Municipal Hospital, Taizhou, Zhejiang, China \\ ${ }^{3}$ Department of Respiratory Medicine, Taizhou Municipal Hospital, Taizhou, Zhejiang, China \\ ${ }^{4}$ Department of Central Laboratory, Taizhou Municipal Hospital, Taizhou, Zhejiang, China \\ ${ }^{5}$ Department of Radiology, the Second Affiliated Hospital, Medical School of Zhejiang University, Hangzhou, Zhejiang, China \\ ${ }^{6}$ Department of Radiology, the First Affiliated Hospital of Wenzhou Medical University, Wenzhou, Zhejiang, China
}

\begin{abstract}
Considering the insufficient understanding of pulmonary cryptococcosis (PC) with consolidation, 22 patients were evaluated based on the chest computed tomography (CT) images and clinical manifestation. The clinical symptoms were mild, mainly manifesting as cough and sputum. Pulmonary lesions mostly involved a single lobe in a single lung with multiple lesions. Specifically, single lung involvement was observed in 17 cases, single lobe in 16 cases and multiple lesions in 14 case. Fifteen cases were mainly distributed in the periphery and 17 cases in the long axis in parallel to the pleura. Nineteen cases had air bronchograms. Eight cases displayed cavitation inside the lesions and 18 cases had surrounding halo signs. Seventeen cases had pleural thickening, of which 10 cases had "pasting wall" signs. The clinical symptoms of PC with consolidation were relatively mild. Comprehensive clinical and imaging performance could improve the diagnosis of the disease.

Keywords: Consolidation, Cryptococcosis, Pulmonary, X-ray computed tomography

Cite this article as: Yang D, Yu L, Luo J, Shen J, Wang D, Kuang P, et al. Characterization of clinical and ct manifestations of pulmonary cryptococcosis with consolidation. Arch Iran Med. 2021;24(6):508-511. doi: 10.34172/aim.2021.73
\end{abstract}

Received: August 17, 2020, Accepted: January 19, 2021, ePublished: June 1, 2021

\section{Introduction}

Pulmonary cryptococcosis (PC) is a pulmonary fungal disease caused by infection with Cryptococcus neoformans or Cryptococcus gattii spores. ${ }^{1,2}$ The incidence of the disease is increasing every year. ${ }^{3,4}$ At present, there remain some difficulties in clinical diagnosis of PC. ${ }^{5}$

The common computed tomography (CT) manifestations of PC include nodules or masses, consolidation, halo sign, ground-glass opacity, and cavitation, among which nodules or masses are the most common manifestations. ${ }^{2,-6} \mathrm{PC}$ with consolidation refers to a mass of solid shadows with unclear boundaries on CT, rather than nodular or mass-like changes, often accompanied by air bronchograms. After reviewing the previous literature, we found that there were few reports on the clinical and imaging manifestations of PC with consolidation other than those addressing PC in general and a lack of systematic analyses. ${ }^{3,6,7} \mathrm{PC}$ with consolidation is mostly observed in immunodeficient or immunocompromised patients, ${ }^{3,7}$ and the clinical manifestation has no specificity. Furthermore, there is no characteristic manifestation of PC with consolidation, and it is difficult to diagnose and identify on CT. Failure to diagnose in a timely manner can easily lead to delayed treatment or unnecessary invasive treatments.

In this study, we retrospectively collected 22 cases of clinically or pathologically confirmed PC with consolidation, and comprehensively analyzed and summarized the clinical and CT manifestations of the lesions to avoid misdiagnosis.

\section{Materials and Methods \\ Clinical Information}

Twenty-two cases of clinically or pathologically confirmed PC with consolidation were collected continuously from September 2013 to June 2020 in our hospital (8 cases), the Second Affiliated Hospital of Medical College of Zhejiang University (6 cases), and the First Affiliated Hospital of Wenzhou Medical University (8 cases). Inclusion criteria: (a) PC with consolidation was shown alone on CT, unaccompanied by nodules or masses, $(b)$ thoracic lesions were improved after antifungal therapy on dynamic follow-up.

Of these, 13 cases were positive for the cryptococcus capsular polysaccharide antigen test, 3 cases were positive for the alveolar lavage fluid, 4 cases were confirmed to be positive after percutaneous lung puncture, and 2 case was determined to be positive after surgical resection. The 
lesions in the patients were resolved by antifungal drugs, except for two case with surgical resection, for which medical treatment was effective.

All patients had detailed medical history and clinical symptoms, which were recorded in outpatient or inpatient cases after careful consultation with the treating clinician. The interval between symptom onset and CT examination in all patients ranged from 2 days to 1 month. The 22 patients comprised 10 males and 12 females aged 21 to 80 years, with a mean age of 51.5 years. None of the patients had a history of raising poultry. There were 13 immunocompetent patients and 9 immunocompromised patients, comprising three cases of diabetes, two cases of chronic hepatitis $\mathrm{B}$, one case of multiple myeloma chemotherapy, one case of systemic lupus erythematosus, one case of Sjogren's syndrome, and one case of chronic renal insufficiency.

\section{Imaging Technique}

Of the 22 patients, 13 underwent plain CT scanning of the chest and 9 underwent plain CT scanning and dual-phase enhanced scanning. A GE Light Speed 16- or 64-row spiral CT machine was used for routine breath-holding training before scanning, and the breath-holding state was calm during the scanning. The scanning range was from the apex of the lungs to the diaphragm, with tube voltage of $120 \mathrm{kV}$, tube current of 120-160 mA, layer thickness of 5-10 $\mathrm{mm}$, and reconstruction layer thickness of 1-2 mm.

\section{Results}

\section{General Patient Information}

All the patients had clinical symptoms, but the symptoms were mild, with chronic or subacute onset. The common symptoms were cough $(19 / 22,86 \%)$, sputum (55\%), fever (32\%), chest pain (18\%) and chest tightness (5\%). The main symptoms were without severe manifestations of hemoptysis, dyspnea, and respiratory failure. Seven patients had fever, five of whom were immunocompromised.

\section{CT Appearances of Lesions}

Among the 22 patients, there were 13 cases (13/22, 59\%) with lesions in the right lung, 5 cases (23\%) in the left and right lobes simultaneously, and 4 case (18\%) in the left lung. There were 16 cases $(73 \%)$ with involvement of a single lobe, comprising 8 cases $(36 \%)$ of the lower lobe of the right lung. There were 6 cases $(27 \%)$ with involvement in two or three lobes. There were 14 cases (64\%) with multiple lesions and 8 cases (36\%) with single lesions. Therefore, in this study, the lung lesions were predominantly located in the right lung and more common in a single lobe, and multiple lesions were present.

Of these cases, 15 of the 22 cases (68\%) had lesions primarily located in the lateral and middle lateral zones. Therefore, the distribution of the lesions was mainly in the peripheral lungs, and the long axis was often parallel to the pleura, which was confirmed in 17 cases. Seventeen cases (77\%) had adjacent pleural thickening and 10 cases
(46\%) had "pasting wall" signs (Figure 1). Nineteen cases (86\%) had air bronchograms in the lesions, of whom 5 cases $(5 / 19,26 \%)$ had bronchus penetration of the lesions, 5 cases $(5 / 19,26 \%)$ had spontaneous termination, and 9 cases $(9 / 19,48 \%)$ had both. Eight cases $(8 / 22,36 \%)$ had cavitation in the lesions, and 18 cases ( $82 \%)$ had halo signs around the lesions.

Other CT signs included pleural effusion (3 cases, 14\%) without calcification, mediastinal lymphadenopathy, and pericardial effusion.

\section{Discussion}

All patients had clinical symptoms in the present study, and this proportion was not consistent with other studies, ${ }^{8,9}$ because all of our patients had PC with consolidation, and patients with nodules or mass-type PC can be asymptomatic. Fever occurred in 32\% (7 cases) with immunocompromised patients accounting for $71 \%$ ( 5 cases) of these cases with fever, which can easily lead to systemic reactions in patients, consistent with a report by Sui et al. ${ }^{3}$ Meanwhile, the present results showed that PC with consolidation changed to a mass of solid shadows, with $64 \%$ (14 cases) having multiple lesions and relatively severe CT manifestations. Therefore, we speculate that the clinical manifestations of PC might not match the imaging manifestations, which needs further verification in large samples.

This study also showed that the lesions were mainly distributed in a single lobe of a single lung, inconsistent with previous PC studies. ${ }^{3,4}$ There were 18 cases involving the right lung and 8 cases involving the left lung, with the right lung being significantly more involved than the left lung. Among 16 cases (73\%) with involvement of a single lobe, 9 cases had involvement of the lower lobe, which was a more common site for lesions than other lobes, consistent with a previous study. ${ }^{10}$ This spatial distribution may be related to the route of respiratory inhalation infection. Cryptococcus spores are more likely to colonize in areas with dense alveoli. The data for this group were mainly for right lung lesions, which were relatively common in the lower lobe.

The lesion distribution in this study mainly involved the peripheral lung, accounting for $68 \%$ of cases, and basically consistent with the report by Xie et $\mathrm{al}^{7}{ }^{7}$ in which $65 \%$ of

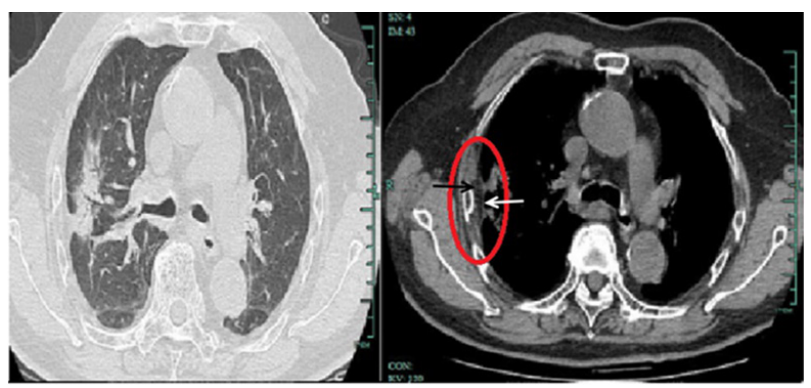

Figure 1. CT showed consolidation in the upper lobe of the right lung, thickening of the adjacent pleura (white arrow in red ellipse), and widening of the extrapleural fat gap, indicating the "pasting wall" sign (black arrow in red ellipse). 
cases had involvement of the peripheral lung. Because of the small diameter of Cryptococcus neoformans spores (1-2 $\mu \mathrm{m})$, these spores are prone to being inhaled and reaching the bronchioles and terminal bronchioles. ${ }^{11}$ The long axis of the lesions was often parallel to the pleura, observed in $77 \%$ of cases. This manifestation reflects the spread and fusion of lesions along alveolar pores, which is common but not specific for PC with consolidation. Halo signs surrounding the lesions, i.e. a shadow of groundglass density surrounding the lesion, were common in this study, found in $82 \%$ of cases. The pathology has been inflammation around a granuloma in previous reports. ${ }^{7,12}$ We concluded that this may be an early stage of the disease, with absorption as the disease progresses. In this study, the most characteristic manifestation was air bronchograms, accounting for $86 \%$, consistent with previous reports. ${ }^{13}$ The bronchus moved through the lesions naturally and smoothly and the distal bronchus gradually narrowed. These signs reflect the inviolability of the corresponding bronchi. In comparison, the air bronchograms of the tumor show the "withered tree sign" or "truncation sign", with the former bronchi appearing stiff and unnatural and the latter proximal bronchi being suddenly interrupted. Another sign in the lesions was cavitation, seen in $36 \%$ of cases. Following the erosion of the bronchioles by cryptococcosis, air can enter the space between granuloma lesions. ${ }^{13}$ In this study, six of these patients were immunocompromised, consistent with the report by $\mathrm{Hu}$ et al. ${ }^{14}$ We found that $\mathrm{PC}$ with consolidation in immunocompromised patients was prone to cavitation in the lesions, while immunocompetent patients tended to show air bronchograms more easily, consistent with other PC studies. ${ }^{9,13}$

The reason for this may be the histopathological types of cryptococcus infection: inflammatory response type and low inflammatory response type..$^{15}$

In this study, adjacent pleural changes occurred in 17 cases, and manifested as pleural thickening, reflecting the characteristics of cryptococcal inflammatory lesions. In 10 cases, the wide base was connected to the pleura, the adjacent pleura was thickened slightly, and the fat space outside the pleura was widened, presenting as a "pasting wall" sign. This sign is presented here and has not been reported in the literature. It might be a common manifestation of inflammatory lesions. On the other hand, tumor lesions are prone to invade the pleura and the fat outside the pleura, resulting in increased density of the fat outside the pleura. This sign was different from the "pleural retraction sign", in which the visceral pleura are pulled in a horn-shaped shadow, do not generally cause pleural thickening, and have the fluid sag content.

Summary of clinical manifestations (Table S1), summary of CT manifestations (Table S2) as well as CTs of a 57-year-old female patient (Figure S1), and 47 (Figure S2) and 49 (Figure S3) years old male patients are presented in Supplementary file 1.

In conclusion, the disease was characterized by peripheral lung and subpleural distribution, with the long axis parallel to the pleura. Air bronchograms and halo signs were the most common CT manifestations of PC with consolidation, especially in immunocompetent patients, whereas cavitation tended to appear in immunocompromised patients. The adjacent pleura was often thickened, and "pasted wall" sign was seen in some cases.

\section{Authors' Contribution}

DY, DW and JS: Study concept and design. LY, JL, PK, and GF: Acquisition of data. DY, PK and GF: Analysis and interpretation of data. DY and DW: Drafting of the manuscript.

\section{Conflict of Interest Disclosures}

There is no conflict of interests for all authors.

Ethical Statement

This research was approved by the Ethics Committee of Municipal Hospital of Taizhou University.

\section{Acknowledgements}

This study was supported by the Medical Health Science and Technology Project of Zhejiang Provincial Health Commission (No. 2019KY784).

\section{Supplementary File}

Supplementary file 1. Summary of clinical and CT manifestations and CTs of a 57-year-old female and 47 and 49 years old male patients.

\section{References}

1. Setianingrum F, Rautemaa-Richardson R, Denning DW. Pulmonary cryptococcosis: a review of pathobiology and clinical aspects. Med Mycol. 2019;57(2):133-50. doi: 10.1093/mmy/myy086.

2. Min J, Huang K, Shi C, Li L, Li F, Zhu T, et al. Pulmonary cryptococcosis: comparison of cryptococcal antigen detection and radiography in Immunocompetent and Immunocompromised patients. BMC Infect Dis. 2020;20(1):91. doi: 10.1186/s12879-020-4818-1.

3. Sui X, Huang Y, Song W, Zheng F, Wang X, Xu X, et al. Clinical features of pulmonary cryptococcosis in thin-section CT in immunocompetent and non-AIDS immunocompromised patients. Radiol Med. 2020;125(1):31-8. doi: 10.1007/ s11547-019-01088-8.

4. Hou X, Kou L, Han X, Zhu R, Song L, Liu T. Pulmonary cryptococcosis characteristics in immunocompetent patients-A 20-year clinical retrospective analysis in China. Mycoses. 2019;62(10):937-44. doi: 10.1111/myc.12966.

5. Gao LW, Jiao AX, Wu XR, Zhao SY, Ma Y, Liu G, et al. Clinical characteristics of disseminated cryptococcosis in previously healthy children in China. BMC Infect Dis. 2017;17(1):359. doi: 10.1186/s12879-017-2450-5.

6. Lindell RM, Hartman TE, Nadrous HF, Ryu JH. Pulmonary cryptococcosis: CT findings in immunocompetent patients. Radiology. 2005;236(1):326-31. doi: 10.1148/ radiol.2361040460.

7. Xie LX, Chen YS, Liu SY, Shi YX. Pulmonary cryptococcosis: comparison of CT findings in immunocompetent and immunocompromised patients. Acta Radiol. 2015;56(4):447-53. doi: 10.1177/0284185114529105. 
8. Deng H, Zhang J, Li J, Wang D, Pan L, Xue X. Clinical features and radiological characteristics of pulmonary cryptococcosis. J Int Med Res. 2018;46(7):2687-95. doi: 10.1177/0300060518769541.

9. Fox DL, Müller NL. Pulmonary cryptococcosis in immunocompetent patients: CT findings in 12 patients. AJR Am J Roentgenol. 2005;185(3):622-6. doi: 10.2214/ ajr.185.3.01850622.

10. He Q, Ding Y, Zhou W, Li H, Zhang M, Shi Y, et al. Clinical features of pulmonary cryptococcosis among patients with different levels of peripheral blood CD4+ T lymphocyte counts. BMC Infect Dis. 2017;17(1):768. doi: 10.1186/ s12879-017-2865-z.

11. Wang D, Wu C, Gao J, Zhao S, MaX, Wei B, et al. Comparative study of primary pulmonary cryptococcosis with multiple nodules or masses by CT and pathology. Exp Ther Med.
2018;16(6):4437-44. doi: 10.3892/etm.2018.6745.

12. Lee YR, Choi YW, Lee KJ, Jeon SC, Park CK, Heo JN. CT halo sign: the spectrum of pulmonary diseases. Br J Radiol. 2005;78(933):862-5. doi: 10.1259/bjr/77712845.

13. Yang R, Yan Y, Wang Y, Liu X, Su X. Plain and contrastenhanced chest computed tomography scan findings of pulmonary cryptococcosis in immunocompetent patients. Exp Ther Med. 2017;14(5):4417-24. doi: 10.3892/ etm.2017.5096.

14. Hu Z, Chen J, Wang J, Xiong Q, Zhong Y, Yang Y, et al. Radiological characteristics of pulmonary cryptococcosis in HIV-infected patients. PLoS One. 2017;12(3):e0173858. doi: 10.1371/journal.pone.0173858.

15. Shibuya K, Hirata A, Omuta J, Sugamata M, Katori S, Saito $\mathrm{N}$, et al. Granuloma and cryptococcosis. J Infect Chemother. 2005;11(3):115-22. doi: 10.1007/s10156-005-0387-x. 